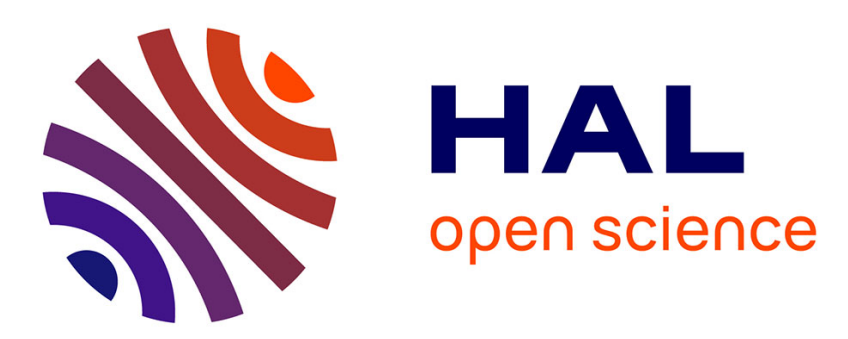

\title{
von Mises-Fisher approximation of multiple scattering process on the hypersphere
}

Florent Chatelain, Nicolas Le Bihan

\section{To cite this version:}

Florent Chatelain, Nicolas Le Bihan. von Mises-Fisher approximation of multiple scattering process on the hypersphere. ICASSP 2013 - 38th IEEE International Conference on Acoustics, Speech and Signal Processing, May 2013, Vancouver, Canada. hal-00865968

\section{HAL Id: hal-00865968 https://hal.science/hal-00865968}

Submitted on 25 Sep 2013

HAL is a multi-disciplinary open access archive for the deposit and dissemination of scientific research documents, whether they are published or not. The documents may come from teaching and research institutions in France or abroad, or from public or private research centers.
L'archive ouverte pluridisciplinaire HAL, est destinée au dépôt et à la diffusion de documents scientifiques de niveau recherche, publiés ou non, émanant des établissements d'enseignement et de recherche français ou étrangers, des laboratoires publics ou privés. 


\title{
VON MISES-FISHER APPROXIMATION OF MULTIPLE SCATTERING PROCESS ON THE HYPERSPHERE
}

\author{
F. Chatelain, N. Le Bihan \\ Gipsa-lab, Images and Signal Department \\ University of Grenoble and Grenoble Institute of Technology, \\ 11 rue des Mathmatiques, Grenoble Campus BP46, 38402 SAINT MARTIN D'HERES, France
}

\begin{abstract}
This paper presents a "method of moments" estimation technique for the study of multiple scattering on the hypersphere. The proposed model is similar to a compound Poisson process evolving on a special manifold: the unit hypersphere. The presented work makes use of an approximation result for multiply convolved von Mises-Fisher distributions on hyperspheres. Comparison with other approximations show the accuracy of the proposed model to provide estimators for the mean free path and concentration parameters when studying a multiple scattering process. Such a process is classically used to model the propagation of waves or particules in random media.
\end{abstract}

Index Terms - von Mises-Fisher distribution, random walk on hypersphere, multiple scattering, Method of moments estimation.

\section{INTRODUCTION}

Mixtures of von Mises-Fisher (vMF) distributions are models used in applications ranging from IRM data analysis [1] to radiation therapy beam direction clustering [2] and speaker clustering [3]. The finite mixture case was originally studied in [4] for data clustering on hyperspheres. All the above mentioned contributions made used of EM algorithms for the estimation of mixtures weights, vMF distribution parameters or number of mixture component. In this paper, we consider particular infinite mixtures of vMFs where the components of the mixtures are multiply convolved vMFs and where the number of components is random, and parameterized by a Poisson parameter $\lambda$.

We consider vMF distributions of unit vector in $\mathbb{R}^{p}$, i.e. elements of $\mathcal{S}^{p-1}$. The proposed approach is thus valid for any dimension $p$. However, our work is motivated by an application in $\mathbb{R}^{3}$. Namely, we consider the problem of multiple scattering for particules in a random media and model the output distribution of the direction of propagation of the particule as a mixture of vMF distribution. This model can be compared with the one proposed in $[5,6]$ where multiple scattering is described as a Compound Poisson Process (CPP) on the rotation group $S O(3)$. In [5], it is shown that this model allows to describe forward multiple scattering, and its accuracy is high when the number of diffusion events is low. Thus, the CPP model describes the behavior of particules in a scattering medium before the fully developed diffusive regime (known to be thoroughly described by the Brownian motion on $S O(3)$ and originally studied by Perrin [7]). Here, we will focus on the multiple scattering regime in its early stage, i.e. when only a few number of scattering events have occurred.

This paper studies a model that mimics the CPP approach, but where the distribution of randomly scattered directions of propaga- tion after $N$ scattering events is obtained thanks to a multiple convolution property of the vMF distribution. Thus, the proposed model can not be expressed using the transitive action of $S O(3)$ on $\mathcal{S}^{2}$, but thanks to the analysis of the distribution of multiply convolved vMF distribution, conditionally to $N$, the parameters of the complete distribution of the multiply scattered particule in a random media can be inferred. The contribution of this paper is to derive an asymptotic (small number of high-concentration scattering events) parametric approximation of the multiply convolved vMF distribution. It allows us to give the Fourier expansion of this approximate distribution. This makes possible the use of a method of moment (MoM) based estimation technique similar to decompounding [6]. We present results of such estimation procedure to identify the parameter of the vMF distribution. The performance of these estimators based on the proposed approximation formula are compared with respect to classical models or approximations used in the literature.

The main contribution of the paper with respect to the existing work is to motivate and provide a vMF approximation for the multiple scattering process on $\mathcal{S}^{p-1}$ in the high concentration, small number of scattering events limit. This approximation, valid for any dimension $p$, offers robust MoM estimators for the inference of vMF mixtures or decompouding.

The remainder of this text is outlined as follows. Properties and asymptotic expression of the random walk distribution on the hypersphere are given in Section 2. Section 3 is devoted to the inference of the compound Poisson process. MoM estimators based on different approximations of the compound Poisson distribution are derived. The accuracy of the asymptotic vMF approximation and the performance of the resulting MoM estimators are analyzed in Section 4 by means of numerical simulation.

\section{SOME PROPERTIES OF THE RANDOM WALK ON THE HYPERSPHERE}

\subsection{General properties}

The $p-1$ hypersphere, denoted as $\mathcal{S}^{p-1}$, is the set of $p$ dimensional vectors with unit length $\mathcal{S}^{p-1}=\left\{\boldsymbol{x} \in \mathbb{R}^{p} ;\|\boldsymbol{x}\|=1\right\}$. The discrete time random walk on the hypersphere $\mathcal{S}^{p-1}$ consists now of a chain of random vectors $\boldsymbol{x}_{0}, \boldsymbol{x}_{1}, \ldots \boldsymbol{x}_{n}$ in $\mathcal{S}^{p-1}$ such that each random step $\boldsymbol{x}_{k-1} \rightarrow \boldsymbol{x}_{k}$, for all $k \geq 1$, are independent. As a consequence the random walk obeys the Markov property since given all the past directions $\boldsymbol{x}_{0}, \ldots, \boldsymbol{x}_{k-1}$, the current direction $\boldsymbol{x}_{k}$ depends only on the previous one $\boldsymbol{x}_{k-1}$. It is of note that these steps are not necessarily identically distributed. However, an important case appears when they are isotropic, so that all the step directions are equiprobable. Thus, the distribution of the $k$ th-step direction $\boldsymbol{x}_{k}$ is rotationally symmetric about the previous one $\boldsymbol{x}_{k-1}$, for all $k \geq 1$. 
When the distribution of the steps are continuous, it yields the following form of the conditional probability density function (pdf) of $\boldsymbol{x}_{k}$ given $\boldsymbol{x}_{k-1}$ [8, p. 179]

$$
f\left(\boldsymbol{x}_{k} \mid \boldsymbol{x}_{k-1}\right) \sim g_{k, k-1}\left(\boldsymbol{x}_{k}^{T} \boldsymbol{x}_{k-1}\right), \quad \forall k \geq 1,
$$

where $\boldsymbol{x}_{k}^{T} \boldsymbol{x}_{k-1}$ is the scalar product of the column vectors $\boldsymbol{x}_{k}$ and $\boldsymbol{x}_{k-1}$. Eq. (1) shows that this conditional distribution does not depend on the part of the vector $\boldsymbol{x}_{k}$ normal to $\boldsymbol{x}_{k-1}$.

Moreover, due to the rotational symmetry, the mean direction of $\boldsymbol{x}_{k}$ given $\boldsymbol{x}_{k-1}$ expresses as

$$
E\left[\boldsymbol{x}_{k} \mid \boldsymbol{x}_{k-1}\right]=\rho_{k, k-1} \boldsymbol{x}_{k-1}, \quad \forall k \geq 1
$$

where the scalar $\rho_{k, k-1} \in(0,1]$ is the so-called mean resultant length. This quantity is directly linked with the dispersion of the directional distribution $g_{k}$. In fact, a value of $\rho_{k, k-1}$ close to 1 indicates a high concentration about the mean direction.

In the remainder of the paper, we assume that the initial direction $\boldsymbol{x}_{0}$ is fixed to a deterministic direction $\boldsymbol{\mu}$. Applying the probability chain rule, the pdf of the $n$th direction can finally be expressed as a multiple integral

$$
\begin{aligned}
f\left(\boldsymbol{x}_{n} ; \boldsymbol{\mu}\right)=\int_{\mathcal{S}^{p-1}} \ldots & \int_{\mathcal{S}^{p-1}} g_{n, 1}\left(\boldsymbol{x}_{n}^{T} \boldsymbol{x}_{n-1}\right) \times \ldots \\
& \times g_{1,0}\left(\boldsymbol{x}_{1}^{T} \boldsymbol{\mu}\right) \boldsymbol{d} \boldsymbol{x}_{n-1} \ldots \boldsymbol{d} \boldsymbol{x}_{1} .
\end{aligned}
$$

Eq. (3) underlines that the pdf of $\boldsymbol{x}_{n}$ can be viewed as the $n$ times multiple convolution on the hypersphere $\mathcal{S}^{p-1}$

$$
f\left(\boldsymbol{x}_{n} ; \boldsymbol{\mu}\right)=\left(g_{n, n-1} \otimes \cdots \otimes g_{1,0}\right)\left(\boldsymbol{x}_{n} ; \boldsymbol{\mu}\right),
$$

where the natural addition operator on Euclidean spaces is replaced by the dot product between unit vectors. In general, it is not possible to obtain a tractable analytical expression of the multiply convolved pdf $(3,4)$. However, some important results can be directly deduced from the Markov property of the random walk. Applying the chain rule on the conditional expectations (2) yields directly the mean value of the random walk.

Proposition 1. For all $n \geq 1$, the mean direction of the $n$-step direction $\boldsymbol{x}_{n}$ and its mean resultant length reads

$$
E\left[\boldsymbol{x}_{n}\right]=\left(\prod_{k=1}^{n} \rho_{k, k-1}\right) \boldsymbol{\mu} .
$$

Proposition 1 shows that the mean direction of the $n$-step is the initial direction $\boldsymbol{\mu}$, while its resultant mean length reduces to the product of all the resultant mean lengths associated with each step. It shows that the directional dispersion increases with the number $n$ of steps, since $\rho_{k, k-1} \leq 1$ for all $k \geq 1$.

Moreover, some well know results in the Euclidean spaces of symmetry and unimodality can be extended to the convolution on the hypersphere.

Proposition 2. Let assume that the conditional directional distributions $f\left(\boldsymbol{x}_{k} \mid \boldsymbol{x}_{k-1}\right)$ are unimodal and rotationally symmetric about their modes (which equals their mean direction) for $k \geq 1$. Then the distribution of the nth-step direction $\boldsymbol{x}_{n}$, whose pdf is given in (3), is also unimodal and rotationally symmetric about its mode. Furthermore, its mode corresponds to the initial direction $\boldsymbol{\mu}$ of the random walk.
Proof. For brevity reason, the proof of the unimodality is omitted. However the sketch of the proof is inspired by the proof given in [9] of the equivalent property on an real line. The proof of the rotational symmetry is given below and is conducted by induction. By hypothesis (1), $\boldsymbol{x}_{1}$ is rotationally symmetric about $\boldsymbol{x}_{0} \equiv \boldsymbol{\mu}$ and the base case holds. We can assume now the inductive hypothesis that $\boldsymbol{x}_{n-1}$ is rotationally symmetric about $\boldsymbol{\mu}$. It leads to the following form of the pdf

$$
f\left(\boldsymbol{x}_{n-1}\right)=h_{n-1}\left(\boldsymbol{x}_{n-1}^{T} \boldsymbol{\mu}\right) .
$$

According to the Bayes' theorem, the marginal density of $\boldsymbol{x}_{n}$ can be derived from its conditional density given $\boldsymbol{x}_{n-1}$ expressed in (1) and from the marginal density of $\boldsymbol{x}_{n-1}$

$$
f\left(\boldsymbol{x}_{n}\right)=\int_{\mathcal{S}^{p}-1}^{g_{n, n-1}}\left(\boldsymbol{x}_{n}^{T} \boldsymbol{x}_{n-1}\right) h_{n-1}\left(\boldsymbol{x}_{n-1}^{T} \boldsymbol{\mu}\right) \boldsymbol{d} \boldsymbol{x}_{n-1} .
$$

In order to prove the rotational symmetry, it is sufficient now to show that for any rotation $R$ defined in the hyperplane normal to the direction $\boldsymbol{\mu}$, the pdf of $\boldsymbol{x}_{n}$ and $\boldsymbol{y}_{n}=R \boldsymbol{x}_{n}$ are equals. Since $R \in S O(p)$ is a rotation matrix, one gets $\operatorname{det}(R)=1, R^{-1}=R^{T}$, $R\left(\mathcal{S}^{p-1}\right)=\mathcal{S}^{p-1}$, and moreover $R \boldsymbol{\mu}=R^{T} \boldsymbol{\mu}=\boldsymbol{\mu}$ as the rotation plane is normal to $\boldsymbol{\mu}$. Thus the pdf of $\boldsymbol{y}_{n}$ is deduced by the change of variable formula as

$$
f\left(\boldsymbol{y}_{n}\right)=\int_{\mathcal{S}^{p}-1}^{g_{n, n-1}}\left(\boldsymbol{y}_{n}^{T} R^{T} \boldsymbol{x}_{n-1}\right) h_{n-1}\left(\boldsymbol{x}_{n-1}^{T} \boldsymbol{\mu}\right) \boldsymbol{d} \boldsymbol{x}_{n-1} .
$$

Performing the change of variable $\boldsymbol{z}=R^{T} \boldsymbol{x}_{n-1}$ for the vector of integration yields

$$
\begin{aligned}
f\left(\boldsymbol{y}_{n}\right) & =\int_{\mathcal{S}^{p-1}} g_{n, n-1}\left(\boldsymbol{y}_{n}^{T} \boldsymbol{z}\right) h_{n-1}\left(\boldsymbol{z}^{T} R^{T} \boldsymbol{\mu}\right) \boldsymbol{d} \boldsymbol{z}, \\
& =\int_{\mathcal{S}^{p-1}}^{g_{n, n-1}}\left(\boldsymbol{y}_{n}^{T} \boldsymbol{z}\right) h_{n-1}\left(\boldsymbol{z}^{T} \boldsymbol{\mu}\right) \boldsymbol{d} \boldsymbol{z}=f\left(\boldsymbol{x}_{n}\right),
\end{aligned}
$$

and the inductive steps holds. It concludes the proof.

\subsection{VMF approximation for isotropic random walk on $\mathcal{S}^{p-1}$}

The main problem to characterize more deeply and to infer the distribution of the $n$ th-step direction $\boldsymbol{x}_{n}$ is that there is no closed form expression of the density of the multiply convolved distribution (4). However when all the random walk steps (1) are governed by unimodal rotationally symmetric distributions, Proposition 2 suggests that the distribution of $\boldsymbol{x}_{n}$ can be well fitted by a standard rotationally symmetric distributions.

The von Mises-Fisher distribution [8, p. 167] is probably the most important distribution in the statistics of hyperspherical data and plays an analogue role on $\mathcal{S}^{p-1}$ than the normal distribution on the real line. This distribution, denoted as $M_{p}(\boldsymbol{\mu}, \kappa)$, is defined by the following pdf for all $\boldsymbol{x} \in \mathcal{S}^{p-1}$

$$
f_{p}(\boldsymbol{x} ; \boldsymbol{\mu}, \kappa)=\frac{\kappa^{p / 2-1}}{(2 \pi)^{p / 2} I_{p / 2-1}(\kappa)} e^{\kappa \boldsymbol{x}^{T} \boldsymbol{\mu}},
$$

where $I_{\nu}(\cdot)$ is the modified Bessel function [10, p. 374], $\boldsymbol{\mu} \in \mathcal{S}^{p-1}$ corresponds to the mean direction and $\kappa \geq 0$ is the concentration parameter: the larger the value of $\kappa$, the more concentrated is the distribution about the mean direction $\boldsymbol{\mu}$. Conversely, when $\kappa=0$ the distribution reduces to the uniform distribution on $\mathcal{S}^{p-1}$. Also, the mean resultant length expresses

$$
\rho \equiv A_{p}(\kappa)=\frac{I_{p / 2}(\kappa)}{I_{p / 2-1}(\kappa)} .
$$


Finally, based on the pdf expression (6), it is straightforward to see that this distribution is rotationally symmetric and unimodal with mode $\boldsymbol{\mu}$ when $\kappa>0$. Since the properties of the vMF distributions are in agreement with Proposition 2, this family seems to be a good candidate to fit the distribution of the $n$th step directions $\boldsymbol{x}_{n}$. By identification of the mean resultant length expressions given in Eq. (5) and (7), it leads to model the distribution of $\boldsymbol{x}_{n}$ by a $M_{p}\left(\boldsymbol{\mu}, \tilde{\kappa}_{n}\right)$ distribution such that

$$
A_{p}\left(\tilde{\kappa}_{n}\right)=\prod_{k=1}^{n} \rho_{k, k-1}
$$

Eq. (8) leads to the vMF approximation of the multiply convolved vMF distribution given in Theorem 1 below.

Theorem 1. Let assume that all the steps are identically distributed according to a $v M F$ distribution with high concentration parameter $\kappa$. Then, based on the Eq. (8) criterion, the asymptotic distribution of $\boldsymbol{x}_{n}$ in the large $\kappa$ and small $n$ asymptotic case is approximated by the $M_{p}\left(\boldsymbol{\mu}, \tilde{\kappa}_{n}\right)$ distribution where

$$
\tilde{\kappa}_{n}=\frac{\kappa-1 / 2}{n}+1 / 2
$$

is the equivalent concentration parameter.

Proof. For large $\kappa$, the asymptotic expansion of the modified Bessel function [10, p. 377] gives the following expansion of eq. (7)

$$
\rho \equiv A_{p}(\kappa)=1+\frac{p-1}{2 \kappa}+\frac{(p-1)(p-3)}{8 \kappa^{2}}+O\left(\frac{1}{\kappa^{3}}\right)
$$

By using the Lagrange inversion theorem, one gets

$$
\kappa=\frac{p-1}{2(1-\rho)}-\frac{p-3}{4}+O(1-\rho),
$$

when $\rho$ is close to 1 . Thus, since $A_{p}\left(\tilde{\kappa}_{n}\right)=\rho^{n}$ according to (8), the expansion of $\tilde{\kappa}_{n}$ is derived by substituting $\rho$ by $\rho^{n}$ in (11)

$$
\tilde{\kappa}_{n}=\frac{p-1}{2\left(1-\rho^{n}\right)}-\frac{p-3}{4}+O\left(1-\rho^{n}\right),
$$

when $\rho$ is close to 1 . Moreover, from (10), one also gets

$\rho^{n}=1-\frac{n(p-1)}{2 \kappa}+\frac{n(p-1)}{2 \kappa}\left[\frac{(p-3)+(n-1)(p-1)}{4 \kappa}\right]+O\left(\frac{1}{\kappa^{3}}\right)$, and reversely

$$
\kappa=\frac{n(p-1)}{2\left(1-\rho^{n}\right)}-\left[\frac{(p-3)+(n-1)(p-1)}{4}\right]+O\left(1-\rho^{n}\right) .
$$

Matching now expression (12) in (13) leads to the formula (9) when $\rho$ is close to 1 , i.e. for large $\kappa$. Note that this formula does not depend anymore on the hyperspherical dimension $p-1$.

\section{MULTIPLE SCATTERING ESTIMATION METHODS}

\subsection{Mixture observation model}

Consider an initial vector $\boldsymbol{x}_{0} \equiv \boldsymbol{\mu} \in \mathcal{S}^{p-1}$. After a time $t$, assume the resulting vector $\boldsymbol{x}_{t} \in \mathcal{S}^{p-1}$ is a mixture made of contributions of rotated versions of $\boldsymbol{\mu}$ an arbitrary number of times $n$, each with individual weight equal to $e^{-\lambda t}(\lambda t)^{n} / n$ !. This weight is obtained when the time interval between two rotation of the vector is chosen to have an exponential distribution with parameter $\lambda$. The equivalent Poisson parameter $\lambda_{t}=\lambda t$ consists in the mean number of rotation events in the elapsed time $t$. In Physics, it is related to the mean free path $\ell$ like $\ell=t / c$ where $c$ is the celerity in the medium. Thus, $\ell$ is the mean distance between two consecutive rotation events (see [5]).

Using the expression of the multi-convolution of $f$ conditioned by the number of rotation events, and recalling the result about the mean direction after $n$ convolutions, one gets:

$$
f_{p}\left(\boldsymbol{x}_{t} ; \boldsymbol{\mu}, \kappa\right)=\sum_{n \geq 0} \frac{e^{-\lambda t}(\lambda t)^{n}}{n !} f_{p}^{\otimes n}(\boldsymbol{\mu}, \kappa),
$$

where $f_{p}^{\otimes n}(\boldsymbol{\mu}, \kappa)$ is the multiply convolved ( $n$ times) vMF distribution. The pdf $f_{p}\left(\boldsymbol{x}_{t} ; \boldsymbol{\mu}, \kappa\right)$ thus consists in a mixture of vMF distributions $n$ time multiply convolved, i.e. $f_{p}^{\otimes n}(\boldsymbol{\mu}, \kappa)=f_{p}\left(\boldsymbol{x}_{n} ; \boldsymbol{\mu}, \kappa\right)$, with $n \in \mathbb{N}$.

\subsubsection{Fourier series expansion}

Harmonic analysis on spheres provides us with the definition of a characteristic function for pdfs taking values on $\mathcal{S}^{p-1}$. In the case of vMF distribution, due to symmetry, the characteristic function takes a simple form [11] as the harmonic basis consists in the Legendre polynomial, i.e.:

$$
f_{p}(\boldsymbol{x} ; \boldsymbol{\mu}, \kappa)=\sum_{\delta \geq 0} N_{p \delta} \hat{f}_{p \delta}(\kappa) P_{p \delta}\left(\boldsymbol{x}^{T} \boldsymbol{\mu}\right),
$$

for $\boldsymbol{x} \in \mathcal{S}^{p-1}$, where $N_{p \delta}$ is a normalization coefficient, $\hat{f}_{p \delta}$ are the Fourier coefficients and $P_{p \delta}\left(\boldsymbol{x}^{T} \boldsymbol{\mu}\right)$ the Legendre polynomials of order $\delta$ in dimension $p$ taken at $\boldsymbol{x}$ with respect to the mean vector $\boldsymbol{\mu}$. The Fourier coefficient of order $p$ is simply obtained by projection of the distribution on the Legendre polynomial:

$$
\widehat{f}_{p \delta}(\kappa)=E\left[P_{p \delta}\left(\boldsymbol{x}^{T} \boldsymbol{\mu}\right)\right]=\frac{I_{\delta+\nu}(\kappa)}{I_{\nu}(\kappa)}
$$

where $\nu=p / 2-1$ [11]. Now, the Fourier expansion of the distribution of $\boldsymbol{x}_{t}$, the resulting vector after a time $t$, can be expressed easily thanks to the approximation property of the multiply convolved vMF given in Theorem 1, i.e. $f_{p}^{\otimes n}(\boldsymbol{x} ; \kappa) \sim f_{p}\left(\boldsymbol{x} ; \tilde{\kappa}_{n}\right)$. Using eq. (14) its Fourier expansion can be written:

$$
f_{p}\left(\boldsymbol{x}_{t} ; \boldsymbol{\mu}, \kappa\right)=\sum_{\delta \geq 0} N_{p \delta} \widehat{f}_{p \delta}^{t}(\kappa) P_{p \delta}\left(\boldsymbol{x}_{t}^{T} \boldsymbol{\mu}\right),
$$

where $\widehat{f}_{p \delta}^{t}(\kappa)$ are the Fourier coefficients, also called Legendre polynomial moments, of $f_{p}\left(\boldsymbol{x}_{t} ; \boldsymbol{\mu}, \kappa\right)$ given by:

$$
\widehat{f}_{p \delta}^{t}(\kappa)=E\left[P_{p \delta}\left(\boldsymbol{x}_{t}^{T} \boldsymbol{\mu}\right)\right]=\sum_{n \geq 0} \frac{e^{-\lambda_{t}}\left(\lambda_{t}\right)^{n}}{n !} \widehat{f}_{p \delta}^{\otimes n}
$$

where $\widehat{f}_{p \delta}^{\otimes n}=\frac{I_{\delta+\nu}\left(\tilde{\kappa}_{n}\right)}{I_{\nu}\left(\tilde{\kappa}_{n}\right)}$ is the Fourier coefficients of the $n$-times convolved vMF $f_{p}\left(\boldsymbol{x} ; \tilde{\kappa}_{n}\right)$. The coefficients $\widehat{f}_{p \delta}^{t}(\kappa)$ are consequently made of infinite series of $\widehat{f}_{p \delta}^{\otimes n}$ which means that they are functions of $\tilde{\kappa}_{n}$ that can be related to $\kappa$ thanks to Theorem 1 .

This makes possible the use of a MoM estimation technique to identify the $\lambda_{t}$ and $\kappa$ parameters from observations of the vector $\boldsymbol{x}_{t}$. 


\subsection{MoM estimation for the compound Poisson vMF}

In order to appreciate the accuracy of the vMF approximation given in Theorem 1, it is useful to compare with classical distribution and approximation used in the directional statistics literature. Since $\kappa$ and $\rho$ are related by a one-to-one transformation (7), the distribution is now parameterized by the parameter vector $\boldsymbol{\theta}=\left(\rho, \lambda_{t}\right)$.

This section addresses the problem of estimating the unknown parameter vector $\boldsymbol{\theta}$ from $N$ independent directions $\boldsymbol{x}_{t}^{1}, \ldots, \boldsymbol{x}_{t}^{N}$ measured at time $t$. This is done by matching the Legendre polynomial moments (15) with their empirical values

$$
\bar{P}_{p \delta}=\frac{1}{N} \sum_{i=1}^{N} P_{p \delta}\left(\boldsymbol{\mu}^{T} \boldsymbol{x}_{t}^{i}\right), \quad \text { for all } \delta \geq 1 .
$$

\subsubsection{VMF approximation}

Based on eq. (15) and using (8), straightforward computations of the Legendre moments yield the following MoM equations

$$
\begin{aligned}
& \bar{P}_{p, 1} \equiv f_{1}\left(\lambda_{t}, \rho\right)=e^{-\lambda_{t}(1-\rho)}, \\
& \bar{P}_{p, 2} \equiv f_{2}\left(\lambda_{t}, \rho\right)=1-p \frac{\rho}{\kappa} \lambda_{t} e^{-\lambda_{t}(1-\rho)} M\left(1,2 \kappa+1, \rho \lambda_{t}\right),
\end{aligned}
$$

with $\kappa=A_{p}^{-1}(\rho)$ and $M(a, b, z)$ is the Kummer function [10, pp. 503-515].

\subsubsection{Hyperspherical Normal approximation}

In the case of large $n$ and $\rho$, the distribution of $\boldsymbol{x}_{t}$ can be approximated by the hyperspherical Normal distribution, corresponding to the Brownian motion on $\mathcal{S}^{p-1}$ [12]. The corresponding Legendre coefficients read

$$
\widehat{f}_{p \delta}^{\otimes n}=\rho^{n \frac{\delta(\delta+p-2)}{2}}, \quad \text { for all } \delta \geq 0, n \geq 1 .
$$

Substituting now $\widehat{f}_{p \delta}^{\otimes n}$ by (17) in the moment expression (15) yields the following MoM equations

$$
\begin{aligned}
& \bar{P}_{p, 1} \equiv g_{1}\left(\lambda_{t}, \rho\right)=e^{-\lambda_{t}(1-\rho)}, \\
& \bar{P}_{p, 2} \equiv g_{2}\left(\lambda_{t}, \rho\right)=e^{-\lambda_{t}\left(1-\rho^{p}\right)} .
\end{aligned}
$$

\subsubsection{Henyey-Greenstein $(H-G)$ approximation}

The Henyey-Greestein function is widely used in numerical simulation of multiple scattering of waves and particules [13]. It is only function of the mean diffusion cosine $E\left[\boldsymbol{x}^{T} \boldsymbol{\mu}\right]$ that can be linked to the heterogeneity parameter of the scatterers in physics applications [5]. Due to rotationally symmetry, the tangent part $\boldsymbol{\mu}^{T} \boldsymbol{x}$ can be modeled according to a H-G distribution. Since,

$$
\widehat{f}_{p \delta}^{\otimes n}=\rho^{n \delta}, \quad \text { for all } \delta \geq 0, n \geq 1,
$$

we obtain the following MoM equations

$$
\begin{aligned}
& \bar{P}_{p, 1} \equiv h_{1}\left(\lambda_{t}, \rho\right)=e^{-\lambda_{t}(1-\rho)}, \\
& \bar{P}_{p, 2} \equiv h_{2}\left(\lambda_{t}, \rho\right)=e^{-\lambda_{t}\left(1-\rho^{2}\right)} .
\end{aligned}
$$

\section{SIMULATION RESULTS}

\subsection{VMF approximation}

To appreciate the accuracy of the vMF approximation given by Theorem 1, Fig. 1 compares the pdf of the random walk tangent part $\boldsymbol{\mu}^{T} \boldsymbol{x}_{n}$ based on the vMF approximation with the real one estimated from $10^{6}$ samples when $n=10$ steps and $p=3$.

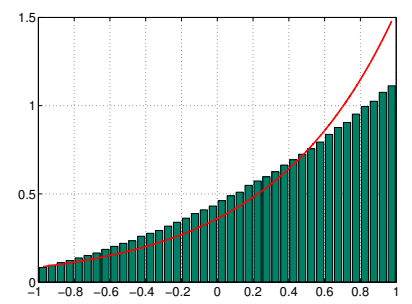

(a) $\rho=0.9(\kappa \approx 10)$

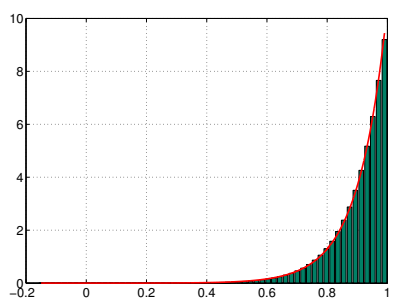

(b) $\rho=0.99(\kappa \approx 100)$
Fig. 1. Green bars: histogram of the tangent part $t=\boldsymbol{x}_{n}^{T} \boldsymbol{\mu}\left(10^{6}\right.$ samples); Red line: pdf of the vMF based approximation $\tilde{t}=\tilde{\boldsymbol{x}}_{n}^{T} \boldsymbol{\mu}$ where $\tilde{\boldsymbol{x}}_{n} \sim M_{p}\left(\boldsymbol{\mu}, \tilde{\kappa}_{n}\right)$

As expected, Fig. 1(a) shows that for low concentration, the asymptotic distribution diverges from the real one. However for high enough concentration, Fig. 1(b) shows the accuracy of the asymptotic approximation.

\subsection{Estimation performance}

These simulations compare the performance of the MoM estimators of $\lambda_{t}$ and $\rho$ corresponding to the vMF, H-G, or hyperspherical normal approximations. They are otained by inverting Eq. (16), (19) and (18) respectively and are referred to as 'VMF', 'H-G' and 'Normal' respectively in the figures. Since there is no closed-form expression of the MoM estimators satisfying (16), the corresponding estimates are numerically evaluated by a Newton-Rhapson like iterative scheme. The number of Monte-Carlo runs is 1000 for all the figures, while the dimension of the vectors is fixed to $p=3$. The mean number of scattering events is $\lambda_{t}=10$ and the mean resultant length of the events is set to $\rho=0.995$ in agreement with the high concentration asymptotic assumption. The MSEs of the considered MoM estimators are depicted and compared on Fig. 2 as a function of the sample size $N$, using a logarithmic scale. Figs. 2(a) and 2(b)

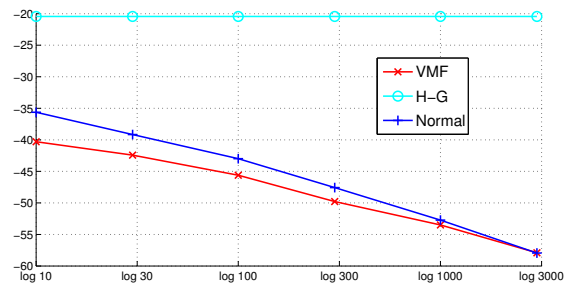

(a) $\widehat{\rho}$ MoM estimators

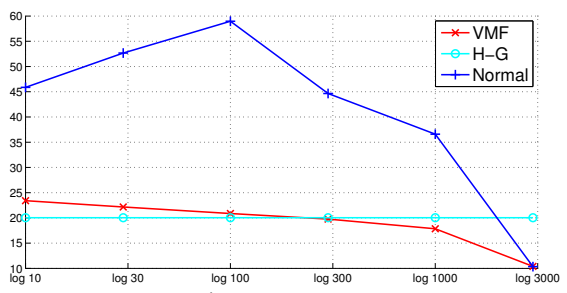

(b) $\widehat{\lambda_{t}}$ MoM estimators

Fig. 2. $10 \log _{10}$ MSEs (dB) vs $\log _{10} N\left(\rho=0.995, \lambda_{t}=10\right)$

emphasize the interest of the vMF based MoM estimators which are more efficient especially for small sample size $N$. It is interesting to note that the H-G approximation yields inconsistant estimates for this highly concentrated multiple vMF scattering case. Finally, these figures show that the vMF approxiamtion yields "reliable" estimates of $\rho$ and $\lambda$ for relatively small sample size. 


\section{REFERENCES}

[1] A. Bhalerao and C.-F. Westin, Hyperspherical von MisesFisher mixture $(\mathrm{HvMF})$ modelling of high angular resolution diffusion MRI, chapter LNCS 4791, pp. 236-243, 2007.

[2] M. Bangert, P. Hennig, and U. Oelfke, "Using an infinite von mises-fisher mixture to cluster treatment beam directions in external radiation therapy," in Proceedings of the Ninth international conference on Machine Learning and applications, 2010, pp. 746-751.

[3] H. Tang and S.M. Chu, "Generative model-based speaker clustering via mixture of von mises-fisher distributions," in Proceedings of the IEEE International Conference on Acoustics, Speech and Signal Processing (ICASSP), 2009, pp. 41014104.

[4] A. Banerjee, I.S. Dhillon, J. Ghosh, and S. Sra, "Clustering on the unit hypersphere using von mises-fisher distributions," Journal of Machine Learning Research, vol. 6, pp. 1345 1382, 2005.

[5] N. Le Bihan and L. Margerin, "Nonparametric estimation of the heterogeneity of a random medium using compound poisson process modeling of wave multiple scattering," Physical Review E, vol. 80, pp. 016601, 2009.

[6] S. Said, C. Lageman, N. Le Bihan, and J.H. Manton, "Decompounding on compact lie groups," IEEE Transactions on Information theory, vol. 56, no. 6, pp. 2766 - 2777, 2010.

[7] F. Perrin, Étude mathématique du mouvement Brownien de rotation, Ph.D. thesis, Faculté des sciences de Paris, 1925.

[8] K.V. Mardia and P.E. Jupp, Directional statistics, John Wiley \& Sons Ltd, 1999.

[9] S. Purkayastha, "Simple proofs of two results on convolutions of unimodal distributions," Statistics \& Probability Letters, vol. 39, no. 2, pp. 97-100, 1998.

[10] M. Abramowitz and I. A. Stegun, Handbook of Mathematical Functions with Formulas, Graphs, and Mathematical Tables, Dover Publications, 1972.

[11] J. Kent, "Limiting behaviour of the von Mises-Fisher distribution," Math. Proc. Camb. Phil. Soc., vol. 84, pp. 531-536, 1978.

[12] P.H. Roberts and H.D. Ursell, "Random walk on a sphere and on a riemannian manifold," Phil. Trans. R. Soc. Lond., A, vol. 252, pp. 317 - 356, 1960.

[13] A. Ishimaru, Wave propagation and scattering in random media, Vol.1,2, Academic Press, New York, 1978. 